\title{
Antibiotic-impregnated polymethylmethacrylate strut graft as a treatment of spinal osteomyelitis: case series and description of novel technique
}

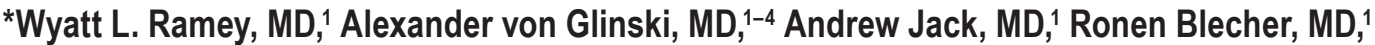 \\ Rod J. Oskouian, MD, ${ }^{1,2}$ and Jens R. Chapman, MD1 \\ ${ }^{1}$ Swedish Neuroscience Institute, Swedish Medical Center, Seattle; ${ }^{2}$ Seattle Science Foundation, Seattle, Washington; \\ ${ }^{3}$ Department of Trauma Surgery, BG University Hospital Bergmannsheil, Ruhr University Bochum, Germany; and ${ }^{4}$ Hansjoerg \\ Wyss Hip and Pelvic Center, Swedish Hospital, Seattle, Washington
}

\begin{abstract}
OBJECTIVE The surgical treatment of osteomyelitis and discitis of the spine often represents a challenging clinical entity for a multitude of reasons, including progression of infection despite debridement, development of spinal deformity and instability, bony destruction, and seeding of hardware. Despite advancement in spinal hardware and implantation techniques, these aforementioned challenges not uncommonly result in treatment failure, especially in instances of heavy disease burden with enough bony endplate destruction as to not allow support of a modern titanium cage implant. While antibiotic-infused polymethylmethacrylate (aPMMA) has been used in orthopedic surgery in joints of the extremities, its use has not been extensively described in the spine literature. Herein, the authors describe for the first time a series of patients treated with a novel surgical technique for the treatment of spinal osteomyelitis and discitis using aPMMA strut grafts with posterior segmental fusion.
\end{abstract}

METHODS Over the course of 3 years, all patients with spinal osteomyelitis and discitis at a single institution were identified and included in the retrospective cohort if they were surgically treated with spinal fusion and implantation of an aPMMA strut graft at the nidus of infection. Basic demographics, surgical techniques, levels treated, complications, and return to the operating room for removal of the aPMMA strut graft and placement of a traditional cage were examined. The surgical technique consisted of performing a discectomy and/or corpectomy at the level of osteomyelitis and discitis followed by placement of aPMMA impregnated with vancomycin and/or tobramycin into the cavity. Depending on the patient's condition during follow-up and other deciding clinical and radiographic factors, the patient may return to the operating room nonurgently for removal of the PMMA spacer and implantation of a permanent cage with allograft to ultimately promote fusion.

RESULTS Fifteen patients were identified who were treated with an aPMMA strut graft for spinal osteomyelitis and discitis. Of these, 9 patients returned to the operating room for aPMMA strut graft removal and insertion of a cage with allograft at an average of 19 weeks following the index procedure. The most common infections were methicillin-sensitive Staphylococcus aureus $(n=6)$ and methicillin-resistant $S$. aureus $(n=5)$. There were 13 lumbosacral infections and 1 each of cervical and thoracic infection. Eleven patients were cured of their infection, while 2 had recurrence of their infection; 2 patients were lost to follow-up. Three patients required unplanned return trips to the operating room, two of which were for wound complications, with the third being for recurrent infection.

CONCLUSIONS In cases of severe infection with considerable bony destruction, insertion of an aPMMA strut graft is a novel technique that should be considered in order to provide strong anterior-column support while directly delivering antibiotics to the infection bed. While the active infection is being treated medically, this structural aPMMA support bridges the time it takes for the patient to be converted from a catabolic to an anabolic state, when it is ultimately safe to perform a definitive, curative fusion surgery.

https://thejns.org/doi/abs/10.3171/2020.3.SPINE191313

KEYWORDS discitis; spondylodiscitis; spinal infection; PMMA; polymethylmethacrylate; management

* W.L.R. and A.v.G. contributed equally to this study. 
$\mathrm{T}$ HE treatment of spinal osteomyelitis and discitis presents a challenging clinical entity within spine surgery. Due to the destructive nature of such severe infections, they may present clinically with any combination of progressive deformity, instability, pain, neurological deficit, or sepsis that may require surgical debridement and fusion. With the advent of modern posterior instrumentation, many have accepted hardware placement in the face of pyogenic spinal osteomyelitis with the main advantages being early ambulation, stabilization, and restoration of sagittal alignment. ${ }^{1-6}$ While structural allografts were more commonly used throughout the 1990s and early 2000s and viewed as safe and effective, interbody cages have also become commonplace in the treatment of vertebral osteomyelitis and discitis, as they provide anterior-column support without the need to obtain autogenous strut graft, which was the preferred method of treatment before titanium struts were commonly used..$^{7-12}$

While the use of posterior fixation alone or in combination with anterior cages provides necessary structural support, there are several potential drawbacks in their utility. Staphylococcus species have been shown to adhere to and create biofilms on various forms of spinal hardware..$^{13,14}$ With this in mind, placing anterior interbody or corpectomy titanium cages into the bed of an active infection poses a risk for recurrence. Although rates of recurrent infection have been shown to be as low as $0 \%-6 \%$ under such circumstances, the surgeon must rely solely on the initial debridement and postoperative intravenous antibiotics for the goal of curing the infection. ${ }^{7,8,12}$ Additionally, in many cases of osteomyelitis and discitis, the endplates are eroded, leaving a raw and tenuous surface for any cage to reliably rest, thereby increasing the risk of hardware failure.

During active severe infection, patients are in a local and global catabolic state. Urgent initial debridement is necessary to begin prompt treatment of the infection with systemic antibiotics, with a delayed staging of fusion when the patient is medically and nutritionally optimized after they have been converted from a catabolic to an anabolic state. ${ }^{9,15}$ However, for reasons previously mentioned, initial placement of a cage and fusion material may not be desirable. Antibiotic-infused polymethylmethacrylate (aPMMA) is a substance that has been frequently used in the field of orthopedics for the treatment of long-bone osteomyelitis, periprosthetic joint infections, and instrumentation infections by providing temporary structural support while directly impregnating the infection bed with antibiotics. ${ }^{16}$ With these factors in mind, we describe for the first time in the spine literature our experience with initially placing aPMMA as a temporary spacer within the osteomyelitis and discitis infection bed along with posterior instrumentation, followed in delayed fashion by removal of the aPMMA strut and placement of a titanium expandable cage and allograft while replacing the posterior hardware with posterolateral fusion. This allows for urgent debridement and temporary structural support after the first stage, followed by a formal fusion when the patient is considerably healthier.

\section{Methods}

Following approval by our institutional review board, we retrospectively reviewed the electronic health records of all adult patients treated at our institution for spinal osteomyelitis, discitis, or epidural abscess. Of these patients who underwent surgical treatment for their infection, those who were treated with placement of an anterior-column aPMMA strut were identified. Basic demographics, location of infection within the spine, necessity for staged fusion, risk factors, and complications were reviewed. All patients had microbial cultures that were collected at the time of initial surgical treatment for their infection. The infectious disease team at our institution then determined the type of intravenous antibiotic(s), dose, and duration of treatment based on culture sensitivities and virulence of the involved pathogen(s).

\section{Surgical Technique}

Once a diagnosis of spinal osteomyelitis and discitis had been clinically established, patients underwent initial posterior surgery for surgical evacuation, debridement, and fixation of their spinal infection (Fig. 1). In the operating room, if there was an abundance of acute pus, evidence of preoperative radiographic instability (such as clear anterolisthesis or lateral listhesis), and destruction of the involved endplates, the decision was made to insert an aPMMA strut graft anteriorly. Prior to doing so, the infection cavity was copiously washed out with a bacitracin-normal saline solution using a thoracoscopic irrigator (Fig. 2). Following this, bone cement manufactured with $1 \mathrm{~g}$ of infused tobramycin was manually mixed with $1 \mathrm{~g}$ of vancomycin powder. One gram of vancomycin is used because it comes packaged in 1-g containers and can be quickly mixed during hardening of the aPMMA. Before setting (hardening) of the aPMMA occurs, it was compacted into the infection bed as much as reasonably possible. Once hardened, the segmental hardware was moderately compressed across the aPMMA strut to ensure a snug fit and correct any local kyphosis. After the infection was felt to be cured with postoperative intravenous antibiotics (at least 6-8 weeks) based on clinical examination findings, laboratory values, and follow-up MRI, patients who were appropriate candidates returned to surgery for replacement of posterior hardware, removal of the aPMMA anterior strut, and placement of an interbody or corpectomy cage with allograft to promote permanent fusion. Antibiotic-infused PMMA removal and replacement with a traditional cage can be performed either posteriorly or laterally in the thoracolumbar regions (Fig. 3).

\section{Results}

Over a 4-year period, 15 patients treated surgically for their vertebral osteomyelitis and discitis underwent implantation of an anterior-column aPMMA strut graft with segmental hardware instrumentation (Table 1). There were 11 males and 4 females with an average age of 64.4 years (range 54-75 years). The most common risk factors for infection included prior surgery, uncontrolled type 2 diabetes mellitus, Charcot arthropathy, and intravenous drug use. The lumbosacral areas of the spine were most often infected, while there was one infection at the cervicothoracic junction and another in the lower thoracic spine. Methicillin-sensitive and methicillin-resistant Staphylococcus aureus were the 2 most common infections. 

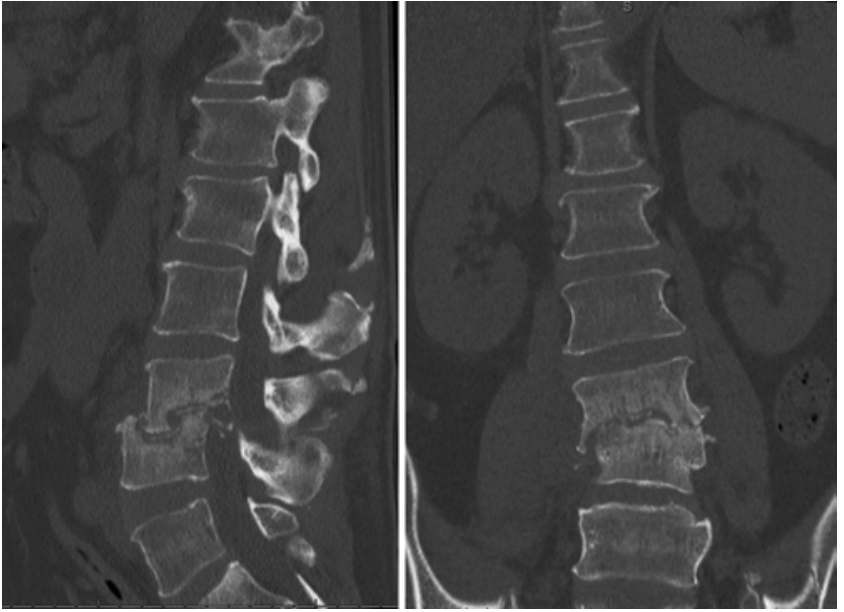

FIG. 1. Case 14. Sagittal (left) and coronal (right) CT scans of the lumbar spine, showing discitis and osteomyelitis at L3-4 after laminectomy and infective spondylolisthesis at L3-4 with severe spinal stenosis and neurological deficit, radiculopathy, and neurogenic claudication.

Nine $(60 \%)$ of 15 patients underwent a second surgery for replacement of the aPMMA strut with a titanium cage and allograft. The average interval between surgeries was 19 weeks (range 2-47 weeks). There were multiple various reasons for not performing a second surgery in the patients who did not have their aPMMA strut removed (Table 1). Of the 9 patients who underwent a second surgery and had adequate radiographic follow-up, all 9 showed radiographic evidence of fusion. Three patients had complications as a result of surgery, which were wound breakdowns and symptomatic seroma formation. Twelve of 13 patients with appropriate follow-up were cured of their infection. One patient with a Charcot arthropathy had recurrent superficial and deep infections following his second-stage surgery, and his infection was not considered cured. Of the other 2 patients with inadequate follow-up, one patient was lost to follow-up and the other underwent a palliative surgery with an aPMMA strut for his sacral infection, after which he died 1 month later of preexisting advanced colon cancer.

\section{Discussion}

The development of aPMMA spacers was based on clinical experience with antibiotic-loaded PMMA beads in septic bone surgery and antibiotic-loaded bone cement in arthroplasty beginning in the $1970 \mathrm{~s} \cdot{ }^{17}$ In spine surgery, PMMA has been used as a cervical disc substitute or in kyphoplasty procedures. ${ }^{18,19}$ Recently, it has been used in stand-alone intervertebral spacers in the lumbar spine. ${ }^{20}$ The present study explored for the first time in the spine literature the use of aPMMA as a temporary spacer within a severe osteomyelitis and discitis infection, followed in delayed fashion by removal of the aPMMA and placement of traditional interbody cages and allograft while replacing the posterior hardware with posterolateral fusion. Twelve of 13 patients in our study with adequate follow-up had their infection successfully eradicated.

The incidence of spinal infections is rising and accounts

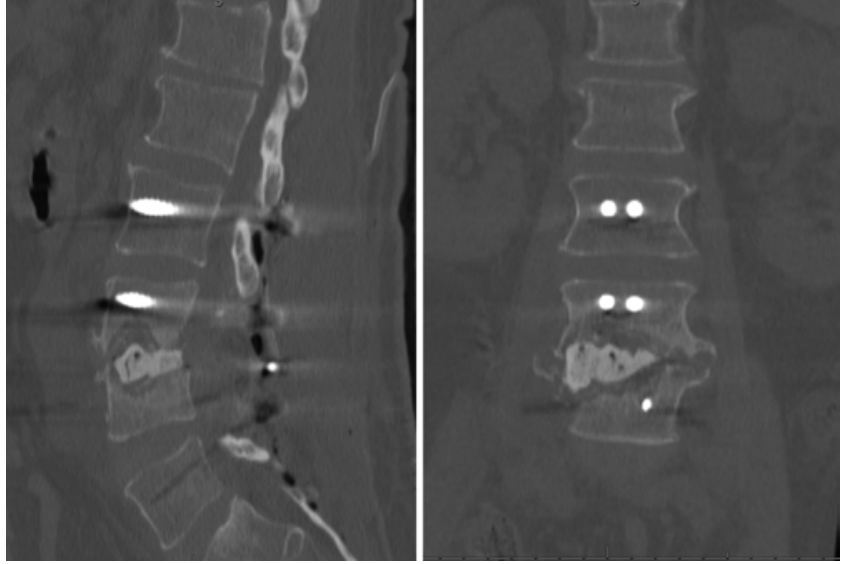

FIG. 2. Case 14. Sagittal (left) and coronal (right) CT scans of the lumbar spine obtained after the first stage of surgery. Decompressive laminectomy was performed at L2-5 with debridement and partial corpectomy of the inferior L3 and superior L4 endplates after pulsatile lavage with $9 \mathrm{~L}$ of a bacitracin-normal saline solution using thoracoscopic irrigation. Intervertebral antibiotic cement spacers (PMMA with tobramycin and vancomycin) were placed, and posterior spinal instrumentation with open reduction of the $\mathrm{L} 2-5$ spondylolisthesis was done.

for about $5 \%$ of osteomyelitis cases. ${ }^{21,22}$ Our cohort reflects that spinal infections are frequently triggered by drug abuse, diabetes, and malnutrition. ${ }^{23}$ Furthermore, we included 3 patients who had Charcot spinal arthropathy. The affected motion segments are at an increased risk of infection, ranging from $14 \%$ to $17 \% .^{24,25}$ Regardless of the osteomyelitis etiology, patients whose conditions are refractory to antibiotic treatment or display neurological deficits should be surgically treated to prevent progressive deformity with vertebral body destruction or spinal instability. ${ }^{26}$
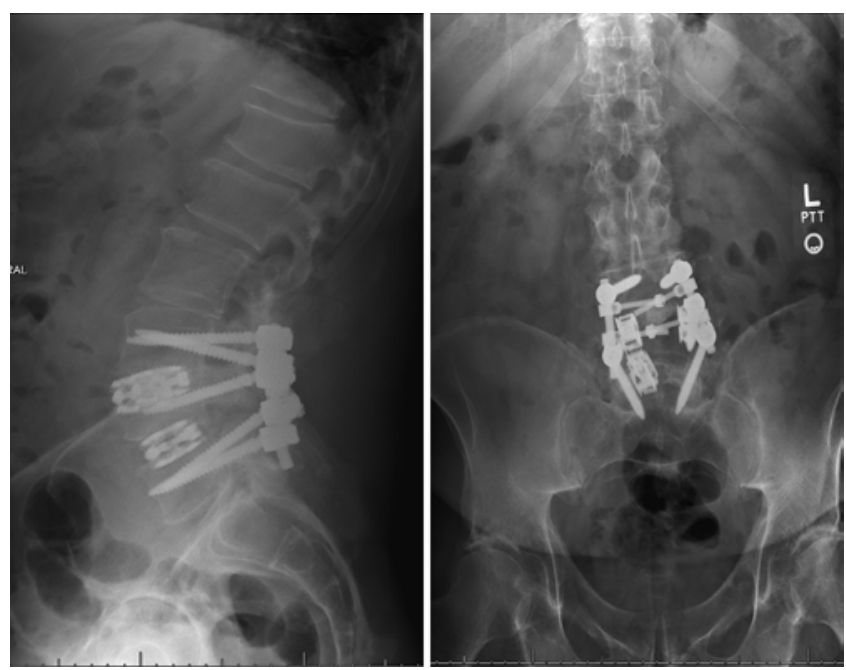

FIG. 3. Case 14. Lateral (left) and anteroposterior (right) radiographs obtained after the second stage of surgery, 3 months after the first procedure. The antibiotic spacers at L3-4 were removed and replaced with posterior lumbar interbody fusion (bilateral expandable cages, bone morphogenetic protein, and local bone graft), and right L4-5 posterior lumbar interbody fusion with unilateral cage and instrumentation from $\mathrm{L} 3$ to $L 5$ with new hardware was placed. 
TABLE 1. Data of patients treated surgically for vertebral osteomyelitis and discitis

\begin{tabular}{|c|c|c|c|c|c|c|c|c|c|}
\hline $\begin{array}{l}\text { Case } \\
\text { No. }\end{array}$ & $\begin{array}{l}\text { Age (yrs), } \\
\text { Sex }\end{array}$ & Level & $\begin{array}{l}\text { 2nd } \\
\text { Op }\end{array}$ & Reason Not Staged & $\begin{array}{l}\text { 2nd-Stage } \\
\text { Approach }\end{array}$ & $\begin{array}{l}\text { Time to Stage } \\
2 \text { Op (wks) }\end{array}$ & Culture & Cure & Risk Factor \\
\hline 1 & $59, \mathrm{M}$ & L3-4 & Yes & & Lateral & 47 & MSSA & Yes & Prior op \\
\hline 2 & $67, \mathrm{M}$ & L3-4 & Yes & & Posterior & 17 & Fungal & Yes & Alcoholism, prior op \\
\hline 3 & $68, M$ & L5-S1 & Yes & & Posterior & 35 & Escherichia coli & Yes & Severe T2DM \\
\hline 4 & $66, F$ & L3-4 & No & Fusion occurred & & & MSSA & Yes & IVDU \\
\hline 5 & $64, \mathrm{M}$ & L2-3 & No & Lost to follow-up & & & MSSA & Yes & Severe T2DM \\
\hline 6 & $68, \mathrm{M}$ & L5-S1 & Yes & & Posterior & 32 & MSSA, Streptococcus viridians & No & Charcot arthropathy \\
\hline 7 & $70, \mathrm{M}$ & T11 & No & Lost to follow-up & & & MRSA & Unk & Severe T2DM \\
\hline 8 & $72, \mathrm{~F}$ & L2-5 & No & Poor nutritional status & & & MSSA, Candida sp. & Yes & Prior op \\
\hline 9 & $58, \mathrm{~F}$ & L5-S1 & No & Refused & & & MRSA & Yes & IVDU \\
\hline 10 & $75, M$ & S2 & No & Palliative & & & Enterobacter cloacae & $\mathrm{No}^{*}$ & Cancer \\
\hline 11 & $58, M$ & L4-5 & Yes & & Posterior & 3 & MRSA & Yes & Charcot arthropathy \\
\hline 12 & $57, \mathrm{M}$ & L2-3 & Yes & & Posterior & 2 & MSSA & Yes & Charcot arthropathy \\
\hline 13 & $54, \mathrm{M}$ & L3-4 & Yes & & Posterior & 19 & Unk & Yes & Prior op \\
\hline 14 & $61, \mathrm{M}$ & C7-T1 & Yes & & $\begin{array}{l}\text { Anterior- } \\
\text { posterior }\end{array}$ & 4 & MRSA & Yes & Prior op \\
\hline 15 & $69, F$ & L3-4 & Yes & & Posterior & 12 & MRSA & Yes & $\begin{array}{l}\text { Morbid obesity, } \\
\text { failure to thrive }\end{array}$ \\
\hline
\end{tabular}

IVDU = intravenous drug use; MRSA = methicillin-resistant Staphylococcus aureus; MSSA= methicillin-sensitive Staphylococcus aureus; T2DM = type 2 diabetes mellitus; unk = unknown.

* Surgery with aPMMA intended to be palliative (patient had end-stage colon cancer).

The main goal of septic surgery should be to remove the infection bed, which acts as a nidus. ${ }^{17,27}$ We presented cases with severe spinal instability and osteomyelitis demanding an extensive debridement, which sacrifices the stability of the anterior column. This combination of severe extensive bony infection requiring radical remediation often requires anterior-column reconstruction. In the case of infection, there is always an uneasiness to operate in an infection bed due to the concern of placing metallic implants and the risk of resulting biofilm on those implants. ${ }^{25,28}$ Bydon et al. retrospectively reported a $17.1 \%$ reoperation rate in 82 patients who underwent debridement and instrumentation..$^{29}$ Arnold and colleagues reviewed 94 patients with vertebral osteomyelitis requiring surgical management with instrumentation and found a $23 \%$ (22 patients) treatment failure rate due to uncontrolled or recurrent infection. Nineteen of these patients underwent further debridement with an average of 2.2 repeat operations, with 15 patients ultimately requiring hardware removal. ${ }^{30}$

Especially in elderly patients or those with multiple comorbidities, the prolonged hospitalization delays mobilization and rehabilitation, and the risk of additional surgery may be a drawback. In severe osteomyelitis in a patient with multiple comorbidities, a 2-stage approach may avoid an extensive primary fusion within an infected bed, and persistent infection due to impaired immunity and biofilm formation on the used cage might be avoided..$^{31}$ The biofilm, comprising colonies of bacteria in a matrix of hydrated polysaccharides, protein, and other molecules, is linked with a decreased metabolism and a lower replication rate of bacteria, resulting in less effectiveness of antimicrobial agents. ${ }^{32,33}$ Implanting aPMMA into the created dead space after bone and soft-tissue debridement (source control) is critical. ${ }^{34}$ Even with antibiotics remaining the cornerstone of pyogenic vertebral osteomyelitis treatment, the penetration of antibiotics in bone defects is low and achieves only low local drug concentrations. ${ }^{35}$ Therefore, aPMMA as local antibiotic delivery systems can release high local concentrations of antibiotic without any associated system toxicity. ${ }^{34}$ Last but not least, the 2-stage approach permits identification of the infecting organism, determination of antibiotic sensitivity, and appropriate adjustment of antibiotic therapy. Even if our 2-stage approach requires a second surgery to remove the aPMMA spacer, patients with multiple morbidities may still benefit from a less invasive primary approach, and it could help to decrease the overall hospital stay and cost due to extensive hardware removal and revision surgeries. ${ }^{30}$

The timing of spacer removal showed large variations between 2 and 47 weeks due to compliance issues (intravenous drug abusers, declined second operation), poor health status of the patient, or a fused spine with the aPMMA spacer in situ and no signs of ongoing inflammation. In general, we recommend spacer removal for several reasons. It is unknown if an aPMMA spine spacer causes bony erosions. García-Oltra et al. did not find any acetabular erosion after aPMMA implantation with a mean aPMMA in situ duration of 48 months ${ }^{36}$ Furthermore, there is an emerging risk of spacer infection over time. Nelson et al. proved a $50 \%(18 / 36)$ rate of positive bacterial culture, which further correlated with recurrent infection following a 2-stage procedure. ${ }^{37}$ The evaluation of spacers regarding the in situ duration is difficult due to numerous factors that might have an influence on the release kinetics in vivo. ${ }^{16}$ Besides the ratio of antibiotics and the type and porosity of the cement, the environmental circumstances might have an ef- 
fect on the release of antibiotics. ${ }^{38}$ Furthermore, most of the knowledge about elution kinetics from aPMMA comes from studies that have investigated its use in cases of hip and knee joint infection. ${ }^{39}$ While aPMMA implantation harbors the above advantages, its removal in the second surgery can be technically challenging due to the abundance of scar tissue that develops and the inherent physical properties of PMMA that make it quite rigid and difficult to remove and release from surrounding tissues. In our experience, we have found that using osteotomes to score the block of aPMMA and removing it piecemeal from any approach (lateral, anterior, or posterior) is the most effective means of its removal.

\section{Limitations}

Most research has focused on 2-stage procedures after periprosthetic infections of the lower limb. Some positive effects might be due to immunomodulation of synovial tissues, which is present in periprosthetic infections (knee and hip) but clearly lacks in cases of spinal osteomyelitis and discitis. ${ }^{40}$ The results presented in this paper are limited by the small numbers and the lack of a control group. Nonetheless, the literature is sparse, and additional trials are urgently needed to improve our understanding and guide treatment decisions.

\section{Conclusions}

The use of aPMMA has been developed and established for treating periprosthetic joint infections. However, there is a lack of evidence and therefore efficient management of protocols in spine infections. Nevertheless, this study reports the successful use of aPMMA in the setting of spinal osteomyelitis and discitis. We have found that an aPMMA spacer in the setting of severely unstable spinal infections provides local antibiotics, reduces dead space, and is able to provide sufficient stability in the setting of radical surgical debridement and stabilization.

\section{References}

1. Carragee EJ. Instrumentation of the infected and unstable spine: a review of 17 cases from the thoracic and lumbar spine with pyogenic infections. J Spinal Disord. 1997;10(4):317-324.

2. Chen WH, Jiang LS, Dai LY. Surgical treatment of pyogenic vertebral osteomyelitis with spinal instrumentation. Eur Spine J. 2007;16(9):1307-1316.

3. Dietze DD Jr, Fessler RG, Jacob RP. Primary reconstruction for spinal infections. J Neurosurg. 1997;86(6):981-989.

4. Hee HT, Majd ME, Holt RT, Pienkowski D. Better treatment of vertebral osteomyelitis using posterior stabilization and titanium mesh cages. J Spinal Disord Tech. 2002;15(2):149-156.

5. Masuda T, Miyamoto K, Hosoe H, et al. Surgical treatment with spinal instrumentation for pyogenic spondylodiscitis due to methicillin-resistant Staphylococcus aureus (MRSA): a report of five cases. Arch Orthop Trauma Surg. 2006;126(5):339-345.

6. Ogden AT, Kaiser MG. Single-stage debridement and instrumentation for pyogenic spinal infections. Neurosurg Focus. 2004;17(6):E5.

7. Liljenqvist $\mathrm{U}$, Lerner T, Bullmann V, et al. Titanium cages in the surgical treatment of severe vertebral osteomyelitis. Eur Spine J. 2003;12(6):606-612.
8. Lu DC, Wang V, Chou D. The use of allograft or autograft and expandable titanium cages for the treatment of vertebral osteomyelitis. Neurosurgery. 2009;64(1):122-130.

9. Nakase H, Tamaki R, Matsuda R, et al. Delayed reconstruction by titanium mesh-bone graft composite in pyogenic spinal infection: a long-term follow-up study. J Spinal Disord Tech. 2006;19(1):48-54.

10. Robertson PA, Rawlinson HJ, Hadlow AT. Radiologic stability of titanium mesh cages for anterior spinal reconstruction following thoracolumbar corpectomy. J Spinal Disord Tech. 2004;17(1):44-52.

11. Schomacher M, Finger T, Koeppen D, et al. Application of titanium and polyetheretherketone cages in the treatment of pyogenic spondylodiscitis. Clin Neurol Neurosurg. 2014;127:65-70.

12. Sundararaj GD, Amritanand R, Venkatesh K, Arockiaraj J. The use of titanium mesh cages in the reconstruction of anterior column defects in active spinal infections: can we rest the crest? Asian Spine J. 2011;5(3):155-161.

13. Chen WH, Jiang LS, Dai LY. Influence of bacteria on spinal implant-centered infection: an in vitro and in vivo experimental comparison between Staphylococcus aureus and Mycobacterium tuberculosis. Spine (Phila Pa 1976). 2011;36(2):103-108.

14. Ha KY, Chung YG, Ryoo SJ. Adherence and biofilm formation of Staphylococcus epidermidis and Mycobacterium tuberculosis on various spinal implants. Spine (Phila Pa 1976). 2005;30(1):38-43.

15. Dimar JR, Carreon LY, Glassman SD, et al. Treatment of pyogenic vertebral osteomyelitis with anterior debridement and fusion followed by delayed posterior spinal fusion. Spine (Phila Pa 1976). 2004;29(3):326-332.

16. Anagnostakos K, Meyer C. Antibiotic elution from hip and knee acrylic bone cement spacers: a systematic review. BioMed Res Int. 2017;2017:4657874.

17. Klemm K, Börner M. Treatment of chronic osteomyelitis with gentamicin PMMA chains. Article in German. Unfallchirurgie. 1986;12(3):128-131.

18. Garfin SR, Yuan HA, Reiley MA. New technologies in spine: kyphoplasty and vertebroplasty for the treatment of painful osteoporotic compression fractures. Spine (Phila Pa 1976). 2001;26(14):1511-1515.

19. Klingler JH, Krüger MT, Sircar R, et al. PEEK cages versus PMMA spacers in anterior cervical discectomy: comparison of fusion, subsidence, sagittal alignment, and clinical outcome with a minimum 1-year follow-up. ScientificWorldJournal. 2014;2014:398396.

20. Varga PP, Jakab G, Bors IB, et al. Experiences with PMMA cement as a stand-alone intervertebral spacer: Percutaneous cement discoplasty in the case of vacuum phenomenon within lumbar intervertebral discs. Orthopade. 2015;44(suppl 1):S1-S7.

21. Sobottke R, Seifert H, Fätkenheuer G, et al. Current diagnosis and treatment of spondylodiscitis. Dtsch Arztebl Int. 2008;105(10):181-187.

22. Zarghooni K, Röllinghoff M, Sobottke R, Eysel P. Treatment of spondylodiscitis. Int Orthop. 2012;36(2):405-411.

23. Blecher R, Yilmaz E, Ishak B, et al. Recent increase in the rate of spinal infections may be related to growing substance-use disorder in the State of Washington: wide population-based analysis of the Comprehensive Hospital Abstract Reporting System (CHARS) database. Spine (Phila Pa 1976). 2019;44(4):291-297.

24. Barrey C, Massourides H, Cotton F, et al. Charcot spine: two new case reports and a systematic review of 109 clinical cases from the literature. Ann Phys Rehabil Med. 2010;53(3):200-220.

25. Jacobs WB, Bransford RJ, Bellabarba C, Chapman JR. Surgical management of Charcot spinal arthropathy: a single- 
center retrospective series highlighting the evolution of management. J Neurosurg Spine. 2012;17(5):422-431.

26. Swanson AN, Pappou IP, Cammisa FP, Girardi FP. Chronic infections of the spine: surgical indications and treatments. Clin Orthop Relat Res. 2006;444(444):100-106.

27. Karthik Yelamarthy PK, Rustagi T, Mahajan R, et al. Infected Charcot spine arthropathy. Spinal Cord Ser Cases. 2018;4:73.

28. Suresh MK, Biswas R, Biswas L. An update on recent developments in the prevention and treatment of Staphylococcus aureus biofilms. Int J Med Microbiol. 2019;309(1):1-12.

29. Bydon M, De la Garza-Ramos R, Macki M, et al. Spinal instrumentation in patients with primary spinal infections does not lead to greater recurrent infection rates: an analysis of 118 cases. World Neurosurg. 2014;82(6):e807-e814.

30. Arnold R, Rock C, Croft L, et al. Factors associated with treatment failure in vertebral osteomyelitis requiring spinal instrumentation. Antimicrob Agents Chemother. 2014;58(2):880-884.

31. Stewart PS. Mechanisms of antibiotic resistance in bacterial biofilms. Int J Med Microbiol. 2002;292(2):107-113.

32. Donlan RM, Costerton JW. Biofilms: survival mechanisms of clinically relevant microorganisms. Clin Microbiol Rev. 2002;15(2):167-193.

33. Patel R. Biofilms and antimicrobial resistance. Clin Orthop Relat Res. 2005;(437):41-47.

34. Cierny G III, DiPasquale D. Treatment of chronic infection. $J$ Am Acad Orthop Surg. 2006;14(10 Spec No.):S105-S110.

35. Ferguson J, Diefenbeck M, McNally M. Ceramic biocomposites as biodegradable antibiotic carriers in the treatment of bone infections. J Bone Jt Infect. 2017;2(1):38-51.

36. García-Oltra E, Bori G, Tomas X, et al. Radiological evaluation of acetabular erosion after antibiotic-impregnated polymethylmethacrylate spacer (Spacer-G). J Arthroplasty. 2013;28(6):1021-1024.

37. Nelson CL, Jones RB, Wingert NC, et al. Sonication of antibiotic spacers predicts failure during two-stage revision for prosthetic knee and hip infections. Clin Orthop Relat Res. 2014;472(7):2208-2214.
38. Anagnostakos K, Kelm J. Enhancement of antibiotic elution from acrylic bone cement. J Biomed Mater Res B Appl Biomater. 2009;90(1):467-475.

39. Penner MJ, Masri BA, Duncan CP. Elution characteristics of vancomycin and tobramycin combined in acrylic bonecement. J Arthroplasty. 1996;11(8):939-944.

40. Singh G, Deutloff N, Maertens N, et al. Articulating polymethylmethacrylate (PMMA) spacers may have an immunomodulating effect on synovial tissue. Bone Joint J. 2016;98B(8):1062-1068.

\section{Disclosures}

The authors report no conflict of interest concerning the materials or methods used in this study or the findings specified in this paper.

\section{Author Contributions}

Conception and design: all authors. Acquisition of data: Ramey, von Glinski, Jack, Blecher. Analysis and interpretation of data: Ramey, von Glinski. Drafting the article: Ramey, von Glinski, Jack, Blecher. Critically revising the article: Ramey, Oskouian, Chapman. Reviewed submitted version of manuscript: Ramey, Jack. Approved the final version of the manuscript on behalf of all authors: Ramey. Statistical analysis: Ramey, von Glinski. Administrative/technical/material support: von Glinski. Study supervision: Oskouian, Chapman.

\section{Supplemental Information}

\section{Previous Presentations}

Portions of this work were presented in poster form at the 17th Annual AOSNA Fellows Forum, Banff Springs, Alberta, Canada, March 28-31, 2019.

\section{Correspondence}

Wyatt L. Ramey: Swedish Neuroscience Institute, Seattle, WA. wyattr12@email.arizona.edu. 